\title{
MÉTODO EXPERIMENTAL PARA DETERMINAÇÃO DAS CAPACITÂNCIAS PARASITAS DO MOTOR DE INDUÇÃO TRIFÁSICO ACIONADO POR INVERSOR MLP
}

\author{
Rudolf Ribeiro Riehl* \\ rrriehlefeb. unesp.br
}

\author{
Ernesto Ruppert ${ }^{\dagger}$ \\ ruppertafee.unicamp.br
}

${ }^{*}$ Depto. de Engenharia Elétrica, Faculdade de Engenharia de Bauru

Universidade Estadual Paulista Julio de Mesquita Filho

Avenida Luiz Edmundo Coube, S/N, 17033-360, Bauru, SP

${ }^{\dagger}$ Depto. de Sistemas e Conversão de Energia

Faculdade de Engenharia Elétrica e Computação, Universidade de Campinas

Avenida Albert Einstein, 400, 13083-370, Campinas, SP

\section{ABSTRACT}

Experimental Method for Determining the Parasitic Capacitances of Three-Phase Induction Motor Driven by PWM Inverter

Three-phase induction motors present stray capacitances. The aim of this paper is to present a new methodology to experimentally determine these capacitances and also evaluates the effects of electromagnetic interference on the motors in common mode. The proposed procedures to the development of this new methodology consists in: a) identification of the motor equivalent electrical circuit parameters through characteristic tests performed in the laboratory; $b$ ) to set up configurations between PWM inverter and the motor for voltage and current measurements: common mode and shaft voltages, leakage and shaft (bearing) currents by using a dedicated measuring circuit; c) to calculate the parasitic capacitance values between stator and frame, stator and rotor, rotor and frame and bearings of the motor using the capacitance characteristic equation; d) use the dedicated software Pspice to simulate the system composed by the three-phase induction motor fed by PWM inverter with the equivalent

Artigo submetido em 10/03/2010 (Id.: 1114)

Revisado em 13/05/2010, 01/10/2010, 24/11/2010, 09/05/2011

Aceito sob recomendação do Editor Associado Prof. Francisco de Assis dos Santos Neves electrical circuit parameters; e) to determine the characteristic waveforms involved in the common mode phenomenon.

KEYWORDS: Three-phase induction motor, PWM inverter, parasitic capacitances.

\section{RESUMO}

A proposta deste trabalho é apresentar uma nova metodologia para determinação experimental das capacitâncias parasitas do motor de indução trifásico de rotor em gaiola. As capacitâncias parasitas fazem parte do circuito equivalente do motor para estudos de interferência eletromagnética causada no motor de indução em modo comum quando ele for acionado por inversor controlado por modulação por largura de pulsos (MLP). Os procedimentos propostos para o desenvolvimento deste novo método consistem em: a) determinação dos parâmetros do circuito equivalente do motor de indução trifásico, em regime permanente, através de ensaio em laboratório; b) estabelecer configurações de ligações entre o inversor MLP e o motor para medições das grandezas de interesse que são as seguintes: tensões de modo comum e de eixo, correntes de fuga e de eixo, através de circuito de medição desenvolvido para este fim; c) calcular os valores das capacitâncias parasitas entre estator e carcaça do motor; estator e rotor; rotor e carcaça e de rolamento utilizando a expressão matemática da 
definição de capacitância; d) utilizar o software Pspice para simular o sistema motor de indução trifásico, alimentado por inversor MLP, com os circuitos equivalentes em baixas e altas frequências; e) obter as formas de onda características do fenômeno de modo comum.

PALAVRAS-CHAVE: Motor de indução trifásico, inversor MLP, capacitâncias parasitas.

\section{INTRODUÇÃO}

A utilização do inversor MLP no acionamento e controle de motores de indução trifásicos é cada vez mais comum, principalmente para a faixa de potência de até $10 \mathrm{cv}$. Com a evolução dos dispositivos de eletrônicos de potência (IGBT, MOSFET e outros), as frequências de chaveamento destes inversores podem chegar a até $20 \mathrm{kHz}$. Nestas frequências, os tempos de subida da tensão MLP são muito pequenos e são responsáveis pelo aparecimento de fenômenos, definidos como interferência eletromagnética no motor de indução, como mostrado na figura 1, Adabi et al. (2007) e Melly (2002). Quanto maiores forem estas frequências de chaveamento, maiores serão as extensões e conseqüências desses fenômenos,Busse et al. (1996) e Akagi and Tamura (2006).

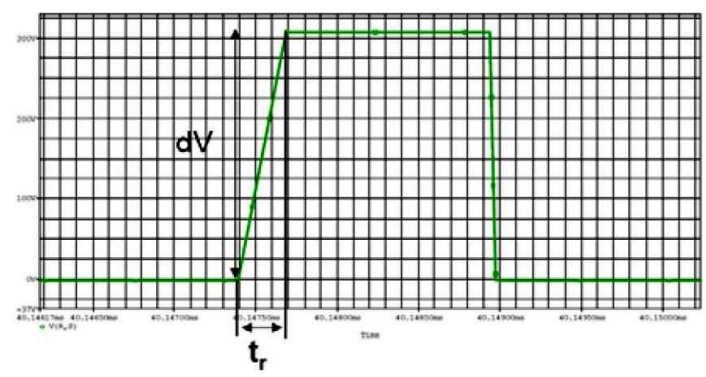

Figura 1: dV/dt da tensão aplicada nas fases do motor.

Devido à presença de capacitâncias parasitas no motor em função de espaços livres ou isolados entre as partes metálicas, ocorrem acoplamentos capacitivos que se tornam caminhos de circulação de correntes elétricas de altas frequências entre as fases do motor (modo diferencial) e entre as fases e o terra (modo comum) são formados, conforme mostrado na figura 2.

Os fenômenos devido ao modo diferencial são responsáveis pelo aquecimento excessivo do motor, prejudicando as características de isolação térmica; rendimento e consequientemente a vida útil do mesmo (queima do motor). Isto ocorre pelo fato do inversor MLP alimentar o motor de indução com uma tensão não senoidal, ou seja, modulada (chaveada), produzindo valores altos de $\mathrm{dV} / \mathrm{dt}$ aplicados nos enrolamentos do estator do motor, Akagi et al. (2004). Os fenômenos devido ao modo comum são responsáveis pelo aparecimento

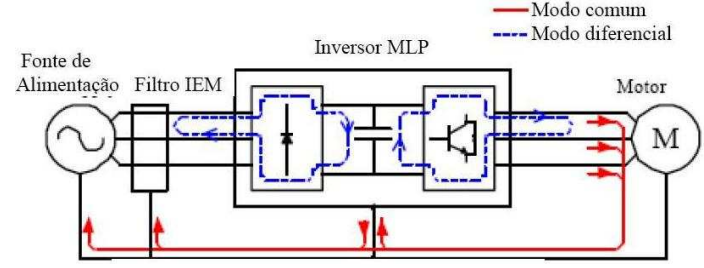

Figura 2: Caminhos de circulação das correntes de alta freqüência.

das correntes entre o motor e o terra que circulam pela carcaça, mancais e pedestais do motor. Como a tensão de modo comum é diferente de zero, é estabelecida uma tensão de eixo entre as partes do mancal e a terra, que é dependente tanto desta tensão de modo comum quanto das capacitâncias parasitas que podem fazer circular correntes elétricas capacitivas por vários caminhos pelo motor, Busse et al. (1996) e Chen et al. (1996). Um destes caminhos passa pelos mancais do motor e as correntes são conhecidas como correntes de mancais (bearing currents) que, em virtude das descargas que ocorrem pelo rompimento do dielétrico (fluído lubrificante do mancal) podem danificar seus rolamentos e provocar o travamento do eixo do motor e consequentemente parada forçada do mesmo podendo ocorrer danos irreversíveis nos rolamentos (figura 3). Outro fenômeno que ocorre é o choque elétrico ou descarga elétrica do motor (DEM), devido à circulação da corrente de fuga $\left(\mathrm{I}_{\mathrm{FUGA}}\right)$ do motor para a carcaça quando o mesmo não está aterrado ou este aterramento não é suficientemente adequado, Busse et al. (1996), Busse et al. (1997) e Charoy and Dunand (2007).

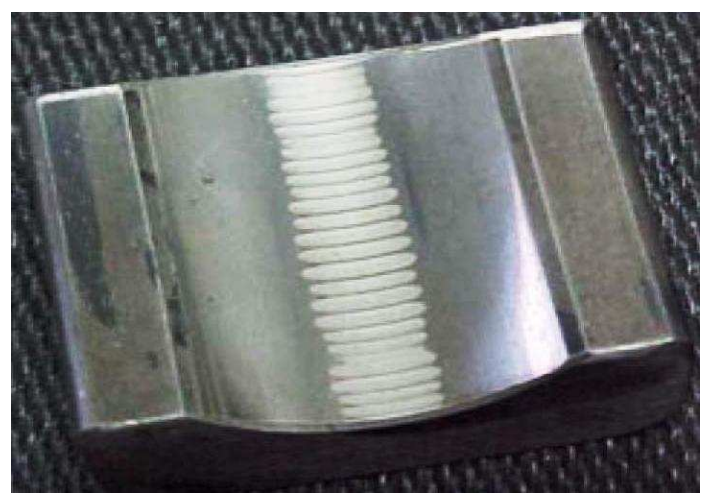

Figura 3: Efeito causado na pista do rolamento.

Neste trabalho foi utilizado um motor de indução trifásico de $5 \mathrm{cv}$, alimentado por um inversor MLP operando com controle volts/hertz. Esta metodologia para determinação de capacitâncias parasitas de motores de indução trifásicos é mostrado em Riehl (2010). Não se tem notícia de Trabalhos Nacionais que utilizam metodologia semelhante para determi- 
nação de capacitâncias parasitas de motores de indução trifásicos.

\section{MÉTODO PARA DETERMINAÇÃO EX- PERIMENTAL DAS CAPACITÂNCIAS PARASITAS DO MOTOR DE INDUÇÃO TRIFÁSICO}

Os procedimentos propostos para o desenvolvimento deste novo método, Riehl (2010), consistem em: a) determinação dos parâmetros do circuito equivalente do motor de indução trifásico, em regime permanente e em alta frequência como é mostrado em Busse et al. (1996), Akagi and Tamura (2006) e Esmaeli et al. (2006), através de ensaio característico em laboratório; b) estabelecer configurações de ligações entre o inversor MLP e o motor para medições das grandezas de interesse: tensões de modo comum $\left(\mathrm{V}_{\mathrm{CM}}\right)$ e de eixo $\left(\mathrm{V}_{\mathrm{EIXO}}\right)$; correntes de fuga $\left(\mathrm{I}_{\mathrm{FUGA}}\right)$ e de eixo ( $\left.\mathrm{I}_{\mathrm{EIXO}}\right)$, através de circuito de medição desenvolvido para este fim, Muetze and Binder (2005) e Chen et al. (1996); c) calcular os valores das capacitâncias parasitas entre estator e carcaça do motor $\left(\mathrm{C}_{\mathrm{EC}}\right)$; estator e rotor $\left(\mathrm{C}_{\mathrm{ER}}\right)$; rotor e carcaça $\left(\mathrm{C}_{\mathrm{RC}}\right)$ e de rolamento $\left(C_{B}\right)$ utilizando suas equações características, (Akagi and Tamura, 2006) e (Esmaeli et al., 2006); d) utilizar o Pspice, para simular o sistema (motor de indução trifásico, alimentado por inversor MLP, com o circuito equivalente em alta frequência do mesmo, Naik et al. (2003) e Arnedo and Venkatesan (2002); e) obter as formas de onda características dos fenômenos de modo comum.

\subsection{Motor de indução trifásico - circuitos equivalentes (CE)}

\subsubsection{CE de baixa frequência (BF) - condição no- minal}

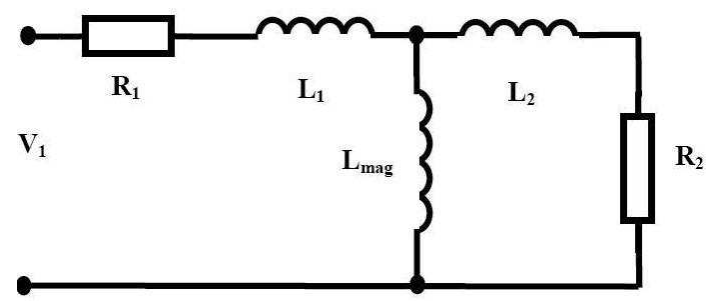

Figura 4: Circuito equivalente de baixa frequência.

Os parâmetros são determinados a partir de ensaios característicos utilizando o método dos dois wattímetros: ensaio em vazio e ensaio com rotor bloqueado, NBR 05383-1 (2002) e, utilizando as equações características apresentadas em Fitzgerald et al. (2003), são determinados os parâmetros do cir- cuito equivalente do motor de indução trifásico de $5 \mathrm{cv}, 4$ pólos, $220 \mathrm{~V}$ e apresentados na tabela 1 .

Tabela 1: Parâmetros do circuito equivalente de BF.

\begin{tabular}{|c|c|c|c|c|}
\hline $\begin{array}{c}\text { Motor } \\
(\mathrm{cv})\end{array}$ & $\mathrm{R}_{1}(\Omega)$ & $\mathrm{R}_{2}(\Omega)$ & $\begin{array}{c}\mathrm{L}_{1}=\mathrm{L}_{2} \\
(\mathrm{mH})\end{array}$ & $\begin{array}{c}L_{\text {mag }} \\
(\mathrm{mH})\end{array}$ \\
\hline 5 & 1.50 & 0.78 & 6.10 & 243.63 \\
\hline
\end{tabular}

\subsubsection{CE de alta frequência - Inversor MLP}

Na figura 5 é mostrado o circuito equivalente do motor de indução trifásico em alta frequência quando alimentado por inversor MLP, Busse et al. (1996) e Esmaeli et al. (2006).

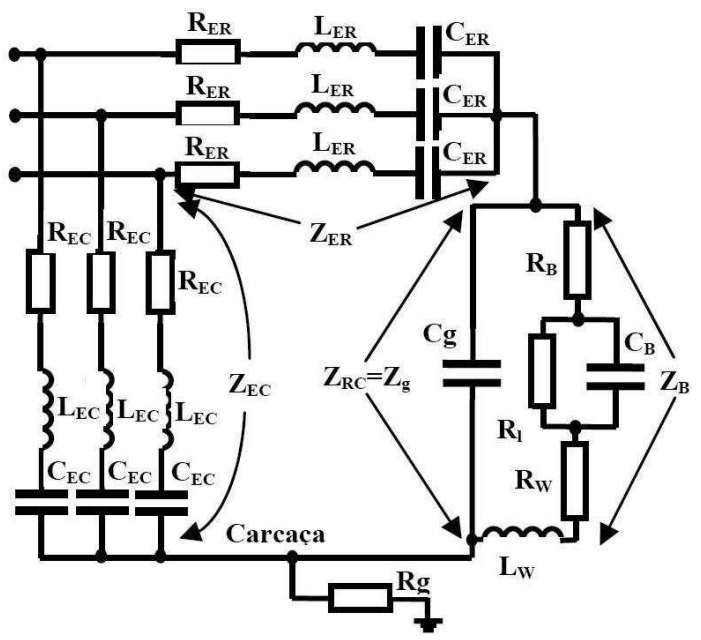

Figura 5: Circuito equivalente de alta frequência do motor.

Simplificações do circuito apresentado na figura 5 podem ser feitas, através das seguintes considerações: a) $\mathrm{Z}_{\mathrm{g}}$ é puramente capacitiva, b) para frequências abaixo de $200 \mathrm{kHz}$ $\mathrm{Z}_{\mathrm{ER}}$ apresenta característica capacitiva e c) $\mathrm{Z}_{\mathrm{RC}}$ apresenta um circuito com comportamento RC. Desta forma, o circuito equivalente simplificado de alta frequência do motor de indução trifásico é mostrado na figura 6.

Nessa figura $\mathrm{V}_{\mathrm{CM}}$ é a tensão de modo comum, $\mathrm{C}_{\mathrm{EC}}$ é a capacitância entre o enrolamento do estator por fase e a carcaça do motor de indução, $\mathrm{C}_{\mathrm{ER}}$ é a capacitância entre os enrolamentos do estator e do rotor, $\mathrm{C}_{\mathrm{RC}}$ é a capacitância entre o rotor e a carcaça e $\mathrm{C}_{\mathrm{B}}$ é a capacitância do rolamento.

Utilizando este circuito equivalente de alta frequência do motor de indução trifásico, são obtidas as equações tanto da tensão de eixo ( $\left.\mathrm{V}_{\mathrm{EIXO}}\right)$ quanto da a corrente de fuga $\left(\mathrm{I}_{\mathrm{FUGA}}\right)$.

$$
\mathrm{V}_{\mathrm{CM}}=\frac{v_{a}+v_{b}+v_{c}}{3}
$$




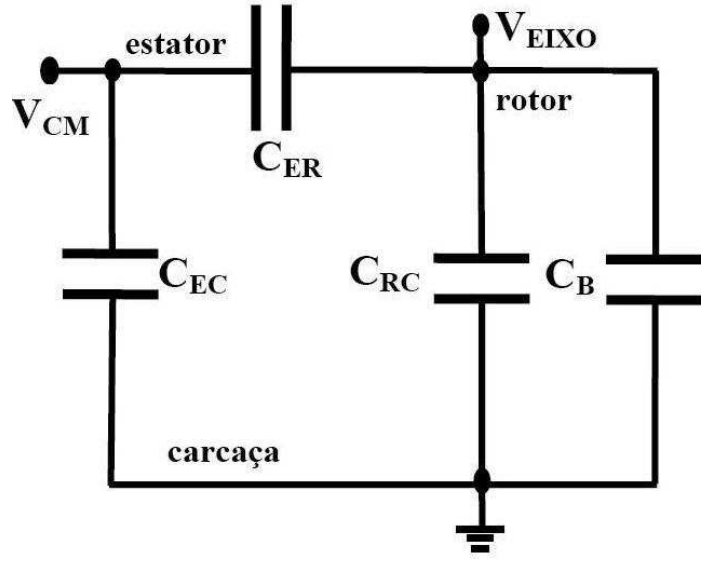

Figura 6: Circuito equivalente de alta frequência do motor.

$$
\begin{aligned}
& \mathrm{V}_{\mathrm{EIXO}}=\mathrm{V}_{\mathrm{CM}}\left(\frac{\mathrm{C}_{\mathrm{ER}}}{\mathrm{C}_{\mathrm{ER}}+\mathrm{C}_{\mathrm{RC}}+\mathrm{C}_{\mathrm{B}}}\right) \\
& \mathrm{I}_{\mathrm{FUGA}}=\frac{\mathrm{V}_{\mathrm{CM}}}{X \mathrm{C}_{\mathrm{EC}}}+\frac{\mathrm{V}_{\mathrm{EIXO}}}{X \mathrm{C}_{\mathrm{RC}}}+\frac{\mathrm{V}_{\mathrm{EIXO}}}{X \mathrm{C}_{\mathrm{B}}}
\end{aligned}
$$

As formas de onda da tensão de eixo $\left(\mathrm{V}_{\mathrm{EIXO}}\right)$ e da corrente de fuga $\left(\mathrm{I}_{\mathrm{FUGA}}\right)$ são mostradas na figura 7.

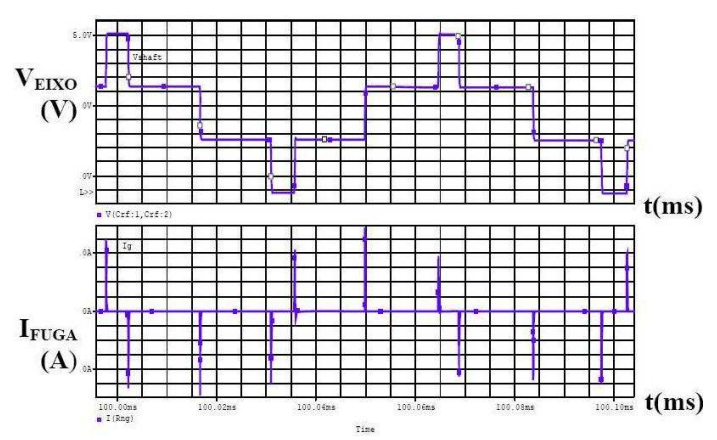

Figura 7: Formas de onda $\mathrm{V}_{\text {eixo }}$ e $\mathrm{I}_{\mathrm{FUGA}}$.

\subsection{Método para determinação experi- mental das capacitâncias parasitas}

Método proposto consiste em se determinar os parâmetros do circuito equivalente de alta frequência do motor de indução trifásico, através de medição direta das grandezas de interesse e, utilizando a equação (4), calcular os valores das capacitâncias. As grandezas de interesse são tensão de modo comum $\left(\mathrm{V}_{\mathrm{CM}}\right)$, tensão de eixo $\left(\mathrm{V}_{\mathrm{EIXX}}\right)$, corrente de fuga $\left(\mathrm{I}_{\mathrm{FUGA}}\right)$ e corrente de eixo ( $\left.\mathrm{I}_{\mathrm{EIXO}}\right)$.

$$
C=\frac{\mathrm{I}_{\mathrm{C}}}{2 \pi f_{S} \mathrm{~V}_{\mathrm{C}}}
$$

Onde: $\mathrm{I}_{\mathrm{C}}$ e $\mathrm{V}_{\mathrm{C}}$ representam a corrente e a tensão eficazes no capacitor, respectivamente e $f_{S}$ a frequência de chaveamento do inversor MLP.

O diagrama esquemático do método proposto é mostrado na figura 8 .

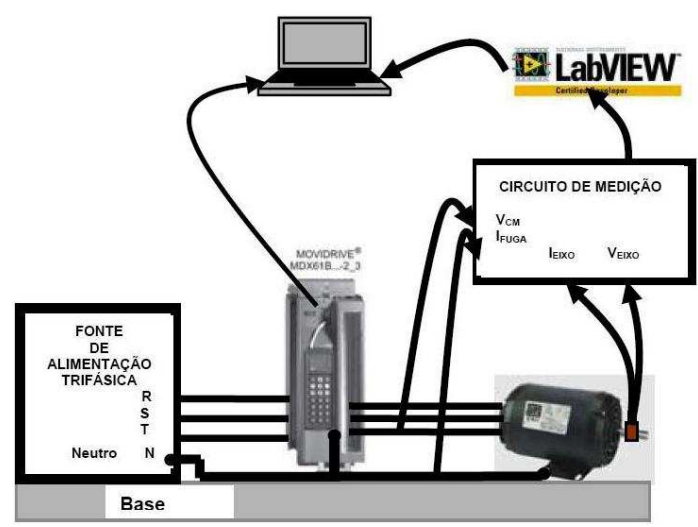

Figura 8: Diagrama de esquemático do método proposto.

A estrutura é constituída pelos seguintes equipamentos: fonte de alimentação trifásica $220 \mathrm{~V} / 60 \mathrm{~Hz}$, Inversor MLP trifásico de $5 \mathrm{cv}$, motor de indução trifásico de $5 \mathrm{cv}$, circuito de medição das grandezas de interesse, placa de aquisição de dados LabView, notebook para gerenciamento do inversor MLP e tratamento dos sinais através de softwares dedicados, base para sustentação dos equipamentos totalmente isolada para permitir que as medições, principalmente das correntes, sejam as mais próximas possíveis das reais, cabo de conexão com o neutro do sistema de alimentação interligado com os terminais de terra do inversor MLP e do motor de indução, proporcionando um caminho de circulação da corrente de fuga do motor.

\subsection{Circuito de medição das grandezas de interesse}

O circuito de medição das grandezas de interesse proposto em Riehl (2010) é mostrado na figura 9.

Estas grandezas são: tensão de modo comum $\left(\mathrm{V}_{\mathrm{CM}}\right)$ : tensão medida do ponto comum (neutro) à carcaça do motor, quando este utiliza conexão estrêla (Y). Quando o mesmo utiliza conexão em delta $(\Delta)$, deve-se fazer uma ligação estrêlaequivalente utilizando-se resistores de alto valor $(1 \mathrm{M} \Omega)$ ligados a cada fase do motor com um ponto em comum (neutro). 


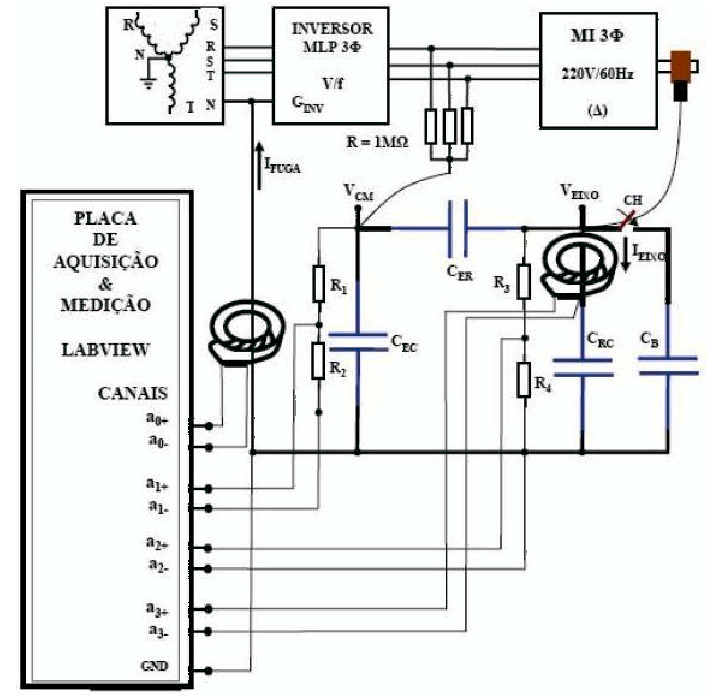

Figura 9: Circuito de medição das grandezas de interesse.

Para a adequação da medição da tensão de modo comum $\left(\mathrm{V}_{\mathrm{CM}}\right)$ à placa de aquisição, adiciona-se um divisor resistivo $\left(\mathrm{R}_{1} ; \mathrm{R}_{2} ; \mathrm{R}_{3}\right.$ e $\left.\mathrm{R}_{4}\right)$ para que a tensão medida não seja superior ao máximo valor permitido pela placa de aquisição (+/- 10V). Para a medição da tensão de eixo ( $\left.\mathrm{V}_{\text {EIXO }}\right)$, que é a tensão medida do eixo à carcaça do motor de indução trifásico, adiciona-se um sistema de anel (cobre)/escôva (carvão) ao eixo do motor de indução. Para a medição da corrente de fuga ( $\mathrm{I}_{\mathrm{FUGA}}$ ), que é a corrente medida no cabo de conexão entre a carcaça do motor de indução e a caixa metálica do inversor MLP, ligados ao neutro do sistema, corrente esta que é composta pela soma das correntes que circulam pelas capacitâncias estator-carcaça $\left(\mathrm{C}_{\mathrm{EC}}\right)$, rotor-carcaça $\left(\mathrm{C}_{\mathrm{RC}}\right)$ e Rolamentos $\left(\mathrm{C}_{\mathrm{B}}\right)$ e, para a medição da corrente de eixo ( $\mathrm{I}_{\mathrm{EIXO}}$ ) que é a corrente medida no condutor que está ligado à escova e que corresponde às contribuições das correntes que circulam pelas capacitâncias rotor-carcaça $\left(\mathrm{C}_{\mathrm{RC}}\right)$ e rolamentos $\left(C_{B}\right)$, utiliza-se sensores de corrente do tipo bobina de Rogowski.

\subsection{Preparação do motor de indução tri- fásico}

A. Os rolamentos do motor são isolados através de uma capa de nylon (tecnil) de alta resistência. Desta forma não haverá circulação de corrente pelos rolamentos (IB) e pode-se avaliar a contribuição do ramo rotor-carcaça, conforme mostrado na figura 10, Akagi and Tamura (2006);

B. Um condutor é ligado entre a superfície externa de um dos rolamentos e o ponto mais próximo à carcaça do motor através de uma chave. Quando a chave esti- ver aberta, tem-se a condição estabelecida no item A. Quando a chave estiver fechada, tem-se então a circulação da corrente do rolamento para a carcaça e retornando para o rolamento, Akagi and Tamura (2006);

C. No eixo do motor é instalado um conjunto polia-escôva, para a medição da tensão de eixo $\left(\mathrm{V}_{\text {EIXO }}\right)$. Utiliza-se também este sistema para se medir a corrente de eixo ( $\mathrm{I}_{\mathrm{EIXO}}$ ), Chen et al. (1996). Na figura 11 são mostrados os itens B e C.

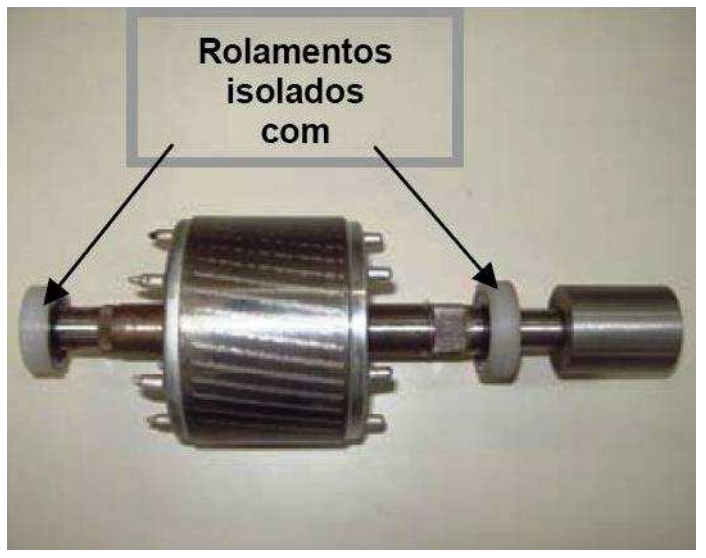

Figura 10: Isolação dos rolamentos do eixo do motor.

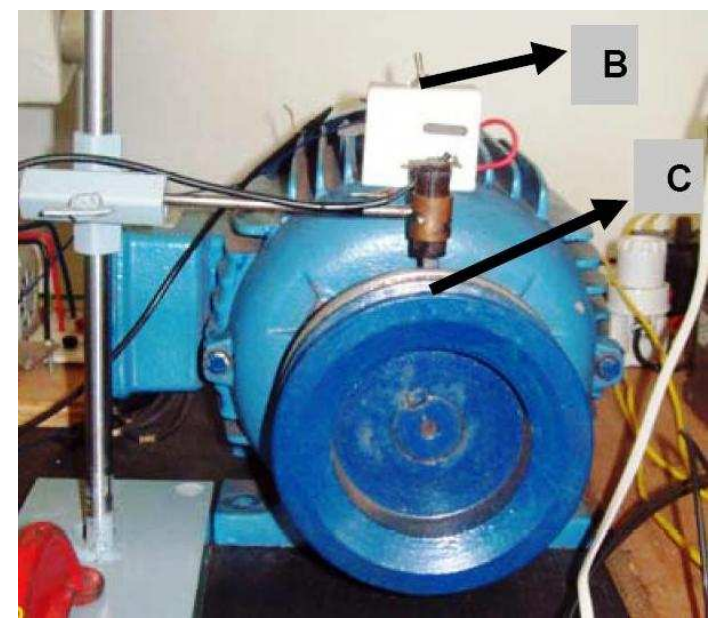

Figura 11: Chave (b) e Conjunto anel-escôva (c) para medição.

\subsection{Placa de aquisição de dados e medi- ção}

Utiliza-se o sistema de medição LabView. A configuração para cada grandeza a ser medida utiliza canais com entradas diferenciais para minimizar os efeitos das tensões de modocomum (ruídos) presentes nas mesmas. São definidos quatro 
canais: canal $a_{0}$ : corrente de fuga - $\mathrm{I}_{\mathrm{FUGA}}$; canal $a_{1}$ : tensão de modo comum - $\mathrm{V}_{\mathrm{CM}}$; canal $a_{2}$ : tensão de eixo - $\mathrm{V}_{\text {EIXO }}$; canal $a_{3}$ : corrente de eixo - I $\mathrm{I}_{\mathrm{EIXO}}$. Através do software dedicado LabView 8.5, cria-se um diagrama de blocos (planta) para o sistema de medição das grandezas de interesse. Na figura 12 mostra-se o diagrama de blocos para corrente de fuga $-I_{F U G A}$.

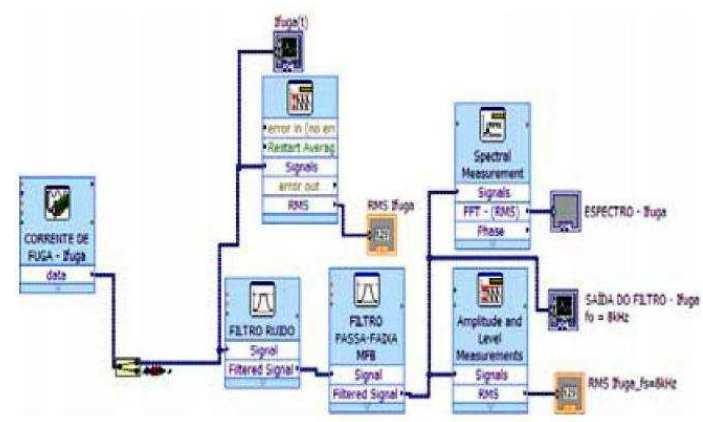

Figura 12: Diagrama de blocos para medição da corrente de fuga.

\subsection{Determinação das capacitâncias pa- rasitas}

O circuito equivalente de alta frequência é novamente mostrado na figura 13. Para a determinação das capacitâncias, deve-se seguir o seguinte procedimento, proposto em Riehl (2010):

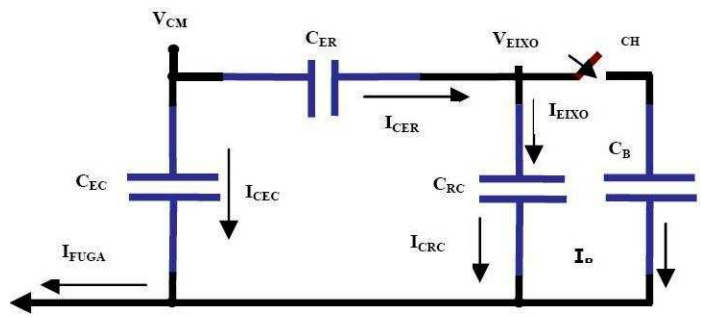

Figura 13: Circuito equivalente de alta frequência.

a. chave $(\mathrm{CH})$ : aberta: os rolamentos do motor estão isolados. Portanto não existe corrente circulando pelos mesmos e a capacitância $C_{B}$ não contribui para o valor de BVR (Bearing Voltage Ratio - Razão da tensão de mancal); b) chave $(\mathrm{CH})$ fechada: passa a circular corrente pelos rolamentos e a capacitância $\mathrm{C}_{B}$ passa a influenciar no valor de BVR. São realizadas as medidas das seguintes grandezas de interesse: tensão de modo comum $\left(\mathrm{V}_{\mathrm{CM}}\right)$; tensão de eixo $\left(\mathrm{V}_{\mathrm{EIXO}}\right)$; corrente de fuga ( $\left.\mathrm{I}_{\mathrm{FUGA}}\right)$; corrente de eixo com a chave $\mathrm{CH}$ desligada ( $\mathrm{I}_{\mathrm{EIXO}-\mathrm{OFF}}$ ); corrente de eixo com a chave $\mathrm{CH}$ ligada
( $\left.\mathrm{I}_{\mathrm{EIXO}-\mathrm{ON}}\right)$. A seguir são determinados os valores das seguintes correntes:

$$
\begin{gathered}
\mathrm{I}_{\mathrm{C}_{\mathrm{EC}}}=\mathrm{I}_{\mathrm{FUGA}}-\mathrm{I}_{\mathrm{EIXO}-\mathrm{OFF}} \\
\mathrm{I}_{\mathrm{C}_{\mathrm{ER}}}=\mathrm{I}_{\mathrm{C}_{\mathrm{RC}}}-\mathrm{I}_{\mathrm{EIXO}-\mathrm{OFF}} \\
\mathrm{I}_{\mathrm{C}_{\mathrm{B}}}=\mathrm{I}_{\mathrm{EIXO}-\mathrm{OFF}}-\mathrm{I}_{\mathrm{EIXO}-\mathrm{ON}}
\end{gathered}
$$

As capacitâncias parasitas do motor de indução trifásico: $\mathrm{C}_{\mathrm{EC}} ; \mathrm{C}_{\mathrm{RC}} ; \mathrm{C}_{\mathrm{ER}}$ e $\mathrm{C}_{\mathrm{B}}$ são determinadas através das equações reescritas abaixo:

$$
\mathrm{C}_{\mathrm{EC}}=\frac{\mathrm{I}_{\mathrm{C}_{\mathrm{EC}}}}{2 \pi f_{S} \mathrm{~V}_{\mathrm{CM}}}
$$

$$
\mathrm{C}_{\mathrm{RC}}=\frac{\mathrm{I}_{\mathrm{CRC}}}{2 \pi f_{S} \mathrm{~V}_{\mathrm{EIXO}}}
$$

$$
\begin{gathered}
\mathrm{C}_{\mathrm{ER}}=\frac{\mathrm{I}_{\mathrm{CER}_{\mathrm{ER}}}}{2 \pi f_{S}\left(\mathrm{~V}_{\mathrm{CM}}-\mathrm{V}_{\mathrm{EIXO}}\right)} \\
\mathrm{C}_{\mathrm{B}}=\frac{\mathrm{I}_{\mathrm{C}_{\mathrm{B}}}}{\mathrm{I}_{\mathrm{C}_{\mathrm{RC}}}} \mathrm{C}_{\mathrm{RC}}
\end{gathered}
$$

\section{ENSAIOS E RESULTADOS COM MO- TOR DE 5 CV}

São realizados ensaios em um motor de indução trifásico de $5 \mathrm{cv}$. O objetivo é o de ratificar que as capacitâncias parasitas dependem apenas das características geométricoconstrutivas do mesmo. Após todas as medições e cálculos, curvas das capacitâncias parasitas são geradas para análise e comparação bem como para a validação do método proposto.Estas curvas são assim estabelecidas: a) capacitâncias $(\mathrm{pF}) \mathrm{x}$ frequência do motor $(\mathrm{Hz})$, para uma frequência de chaveamento fixa; b) capacitâncias $(\mathrm{pF}) \mathrm{x}$ frequência de chaveamento $(\mathrm{kHz})$, para a frequência do motor de $60 \mathrm{~Hz}$; c) valor médio das capacitâncias $(\mathrm{pF}) \mathrm{x}$ frequência de chaveamento $(\mathrm{kHz})$. Formas de ondas das grandezas de interesse também foram obtidas através de medição com osciloscópio digital.

As tabelas 2-4 apresentam: as medições das grandezas de interesse (Tabela 2), os valores calculados das correntes que circulam entre o estator e a carcaça do motor $\left(\mathrm{I}_{\mathrm{C}_{\mathrm{EC}}}\right)$ e nos rolamentos $\left(\mathrm{I}_{\mathrm{C}_{\mathrm{B}}}\right)$ (Tabela 3 ) e valores calculados das capacitâncias parasitas do motor de indução trifásico de 5 cv (Tabela 4). 
Tabela 2: Medições das grandezas de interesse.

\begin{tabular}{|l|l|l|l|l|l|l|}
\hline $\begin{array}{l}\text { Frequência de chaveamento do } \\
\text { inversor }(\mathrm{kHz})\end{array}$ & $\begin{array}{l}\text { Frequência do } \\
\text { motor }(\mathrm{Hz})\end{array}$ & $\mathrm{V}_{\mathrm{CM}}(\mathrm{V})$ & $\mathrm{V}_{\text {EIXO }}(\mathrm{V})$ & $\begin{array}{l}\mathrm{I}_{\mathrm{FUGA}} \\
(\mathrm{mA})\end{array}$ & $\begin{array}{l}\mathrm{I}_{\text {EIXO }}\left(\mathrm{ch}_{\text {off }}\right) \\
(\mathrm{mA})\end{array}$ & $\begin{array}{l}\mathrm{I}_{\text {EIXO }}\left(\mathrm{ch}_{\text {on }}\right) \\
(\mathrm{mA})\end{array}$ \\
\hline 4 & 20 & 112,58 & 3,92 & 12,60 & 0,160 & 0,098 \\
\hline & 30 & 99,21 & 3,45 & 11,30 & 0,141 & 0,091 \\
\hline & 40 & 83,04 & 2,88 & 9,95 & 0,119 & 0,081 \\
\hline & 50 & 63,44 & 2,18 & 7,70 & 0,090 & 0,078 \\
\hline 8 & 60 & 42,30 & 1,44 & 6,30 & 0,061 & 0,054 \\
\hline & 20 & 106,05 & 3,78 & 24,00 & 0,243 & 0,148 \\
\hline & 30 & 94,23 & 3,34 & 21,50 & 0,215 & 0,139 \\
\hline & 40 & 79,00 & 2,77 & 18,10 & 0,178 & 0,121 \\
\hline & 50 & 60,33 & 2,13 & 14,30 & 0,139 & 0,120 \\
\hline 12 & 60 & 39,81 & 1,39 & 9,65 & 0,091 & 0,080 \\
\hline & 20 & 105,12 & 3,78 & 36,50 & 0,356 & 0,217 \\
\hline & 30 & 93,30 & 3,33 & 32,90 & 0,319 & 0,206 \\
\hline & 40 & 77,75 & 2,75 & 26,90 & 0,261 & 0,178 \\
\hline & 50 & 58,47 & 2,08 & 21,00 & 0,199 & 0,171 \\
\hline 16 & 37,63 & 1,37 & 13,50 & 0,128 & 0,112 \\
\hline & 60 & 102,94 & 3,74 & 49,30 & 0,485 & 0,295 \\
\hline & 20 & 91,43 & 3,25 & 43,30 & 0,405 & 0,262 \\
\hline & 30 & 74,95 & 2,68 & 35,40 & 0,342 & 0,233 \\
\hline & 40 & 57,54 & 2,02 & 27,08 & 0,260 & 0,224 \\
\hline & 50 & 37,94 & 1,38 & 18,25 & 0,173 & 0,152 \\
\hline
\end{tabular}

Tabela 3: Correntes $\mathrm{I}_{\mathrm{C}_{\mathrm{EC}}}$ e $\mathrm{I}_{\mathrm{C}_{\mathrm{B}}}$ calculadas.

\begin{tabular}{|l|l|l|l|}
\hline $\begin{array}{l}\text { Frequência de } \\
\text { chaveamento do } \\
\text { inversor }(\mathrm{kHz})\end{array}$ & $\begin{array}{l}\text { Frequência do } \\
\text { motor }(\mathrm{Hz})\end{array}$ & $\begin{array}{l}\mathrm{I}_{\mathrm{C}_{\mathrm{EC}}} \\
(\mathrm{mA})\end{array}$ & $\begin{array}{l}\mathrm{I}_{\mathrm{C}_{\mathrm{B}}} \\
(\mathrm{mA})\end{array}$ \\
\hline 4 & 20 & 12,44 & 0,062 \\
\hline & 30 & 11,16 & 0,050 \\
\hline & 40 & 9,83 & 0,038 \\
\hline & 50 & 7,61 & 0,012 \\
\hline & 60 & 6,24 & 0,007 \\
\hline 8 & 20 & 23,76 & 0,095 \\
\hline & 30 & 21,29 & 0,076 \\
\hline & 40 & 17,92 & 0,057 \\
\hline & 50 & 14,16 & 0,019 \\
\hline & 60 & 9,56 & 0,011 \\
\hline 12 & 20 & 36,14 & 0,139 \\
\hline & 30 & 32,58 & 0,113 \\
\hline & 40 & 26,64 & 0,083 \\
\hline & 50 & 20,80 & 0,028 \\
\hline & 60 & 13,37 & 0,016 \\
\hline 16 & 20 & 48,82 & 0,190 \\
\hline & 30 & 42,90 & 0,143 \\
\hline & 40 & 35,06 & 0,109 \\
\hline & 50 & 26,82 & 0,036 \\
\hline & 60 & 18,08 & 0,021 \\
\hline
\end{tabular}

As figuras a seguir mostram as curvas das capacitâncias parasitas do motor de $5 \mathrm{cv}$. Nas figuras 14 são mostradas as características das capacitâncias parasitas em função da frequência do motor (velocidade), para frequências de chaveamento específicas do inversor de $4 \mathrm{kHz}, 8 \mathrm{kHz}, 12 \mathrm{kHz}$ e $16 \mathrm{kHz}$ ).

Na figura 15, apresenta-se os valores das capacitâncias parasitas em função da frequência de chaveamento do inversor MLP, para a frequência do motor de $60 \mathrm{~Hz}$. Nota-se novamente que as mesmas mantém a sua linearidade, confirmando a dependência exclusiva as características físicoconstrutivas.

Na figura 16, são apresentados os valores médios das capacitâncias parasitas do motor de $5 \mathrm{cv}$.

\section{SIMULAÇÕES USANDO O PSPICE E MEDIÇÕES}

O sofware Pspice foi utilizado para simular o método proposto e comparar as formas de onda obtidas nas simulações com as medições reais feitas utilizando osciloscópio digital. O circuito esquemático de simulação pode ser visto no Apêndice A. As formas de onda (a) são medidas através de osciloscópio digital. As formas de onda (b) são obtidas através de simulação usando Pspice (figura A.1). Observar que, na simulação, são utilizados componentes ideais de chaves semicondutoras. As formas de onda mostradas a seguir são das 
Tabela 4: Capacitâncias parasitas calculadas.

\begin{tabular}{|l|l|l|l|l|l|}
\hline $\begin{array}{l}\text { Frequência de chaveamento do } \\
\text { inversor }(\mathrm{kHz})\end{array}$ & $\begin{array}{l}\text { Frequência do } \\
\text { motor }(\mathrm{Hz})\end{array}$ & $\mathrm{C}_{\mathrm{EC}}(\mathrm{pF})$ & $\mathrm{C}_{\mathrm{RC}}(\mathrm{pF})$ & $\mathrm{C}_{\mathrm{ER}}(\mathrm{pF})$ & $\mathrm{C}_{\mathrm{B}}(\mathrm{pF})$ \\
\hline 4 & 20 & 4396,75 & 1624,08 & 58,09 & 1027,48 \\
\hline & 30 & 4475,51 & 1626,20 & 58,59 & 893,51 \\
\hline & 40 & 4710,68 & 1644,10 & 59,07 & 771,30 \\
\hline & 50 & 4773,03 & 1642,70 & 58,46 & 252,72 \\
\hline & 60 & 5868,78 & 1658,54 & 59,40 & 218,50 \\
\hline 8 & 20 & 4456,81 & 1278,96 & 47,27 & 820,95 \\
\hline & 30 & 4493,94 & 1280,66 & 47,06 & 700,22 \\
\hline & 40 & 4513,38 & 1278,45 & 46,46 & 602,24 \\
\hline & 50 & 4669,86 & 1298,31 & 47,52 & 205,57 \\
\hline & 60 & 4777,09 & 1302,48 & 47,12 & 179,09 \\
\hline 12 & 20 & 4560,40 & 1249,14 & 46,59 & 800,14 \\
\hline & 30 & 4631,64 & 1270,57 & 47,03 & 696,96 \\
\hline & 40 & 4544,32 & 1258,81 & 46,16 & 586,97 \\
\hline & 50 & 4718,49 & 1268,94 & 46,81 & 207,78 \\
\hline & 60 & 4713,18 & 1329,20 & 46,82 & 177,03 \\
\hline 16 & 20 & 4717,18 & 1289,98 & 48,63 & 830,83 \\
\hline & 30 & 4666,93 & 1239,61 & 45,69 & 676,58 \\
\hline & 40 & 4652,95 & 1269,42 & 47,07 & 593,85 \\
\hline & 50 & 4636,62 & 1280,37 & 46,58 & 205,77 \\
\hline & 60 & 4739,60 & 1247,04 & 47,07 & 172,29 \\
\hline
\end{tabular}

grandezas de interesse com frequência de chavemento do inversor MLP de $16 \mathrm{kHz}$ e frequência do motor de $60 \mathrm{~Hz}$. As figuras 17(a) e 17(b) apresentam as formas de onda das tensões de modo comum $\left(\mathrm{V}_{\mathrm{CM}}\right)$ e de eixo $\left(\mathrm{V}_{\mathrm{EIXO}}\right)$.

As figuras 18(a) e 18(b) apresentam as formas de onda das tensões de modo comum $\left(\mathrm{V}_{\mathrm{CM}}\right)$ e da corrente de fuga ( $\left.\mathrm{I}_{\mathrm{FUGA}}\right)$.

Na comparação das formas de onda acima, percebe-se que apesar de na simulação serem utilizadas características ideais do sistema, o comportamento das mesmas são muito semelhantes. Assim, uma vez determinadas as capacitâncias parasitas do motor de indução trifásico de rotor em gaiola, a simulação pode ser utilizada para análises em diversas condições de operação do motor e também otimizar o desenvolvimento de projeto de filtros para redução dos fenômenos de interferência eletromagnética.

\section{CONCLUSÕES}

A metodologia proposta, para a determinação das capacitâncias parasitas do motor de indução trifásico, quando acionado por inversor MLP, apresenta resultados consistentes e coerentes. Os resultados dos ensaios realizados permitem afirmar que as capacitâncias parasitas do motor de indução trifásico, são função apenas das características geométricoconstrutivas do mesmo. No ensaio do motor de indução tri- fásico de $5 \mathrm{cv}$, foram obtidos os seguintes valores médios das capacitâncias parasitas: $\mathrm{C}_{\mathrm{EC}}=4682 \mathrm{pF} ; \mathrm{C}_{\mathrm{RC}}=1265 \mathrm{pF}$; $\mathrm{C}_{\mathrm{ER}}=141 \mathrm{pF}$ e $\mathrm{C}_{\mathrm{B}}=495 \mathrm{pF}$. Comparando estes valores com os apresentados por Busse et al. (1996), Busse et al. (1997) e Charoy and Dunand (2007), para a mesma potência, têm-se resultados muito próximos, conforme mostrados nas figuras 19 e 20.

As variações da frequência de chaveamento alteram pouco o valor das capacitâncias. O que tem seus valores alterados são as reatâncias capacitivas correspondentes às mesmas devido às frequências das harmônicas. $\mathrm{O}$ aumento da frequência de chaveamento do inversor MLP, apesar de melhorar a característica da forma de onda da corrente de carga, faz com os tempos de chaveamentos dos dispositivos de potência (IGBT ou MOSFET) sejam bastante reduzidos, implicando no aumento das taxas de crescimento da tensão (dV/dt). Isto reflete diretamente nas correntes que circulam pelas capacitâncias parasitas e consequentemente na corrente de fuga. Também ocorre o aumento das amplitudes destas correntes em razão de que as reatâncias capacitivas parasitas são sensivelmente diminuídas em seu valor, devido ao aumento da frequência de chaveamento. Com isto aumentam-se os efeitos de interferência eletromagnética tanto nos rolamentos (correntes de rolamento) quanto nas correntes capacitivas circulantes no motor. Observa-se também que os valores das capacitâncias de rolamento $\left(\mathrm{C}_{\mathrm{B}}\right)$ diminuem com o aumento da velocidade do motor de indução, Akagi et al. (2004). 


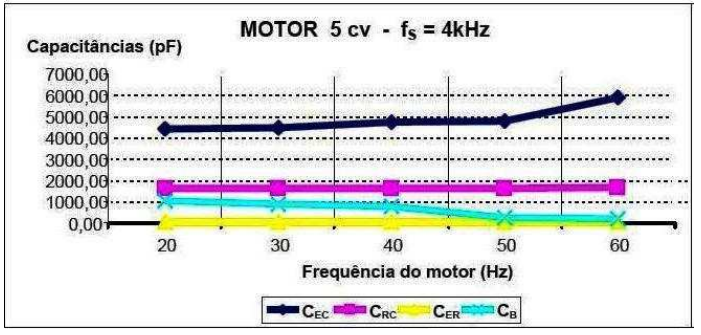

(a)

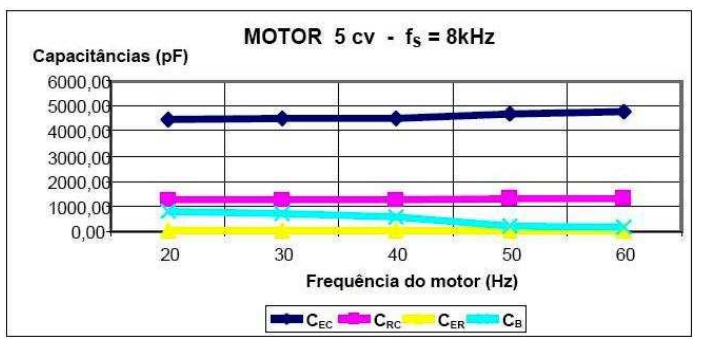

(b)

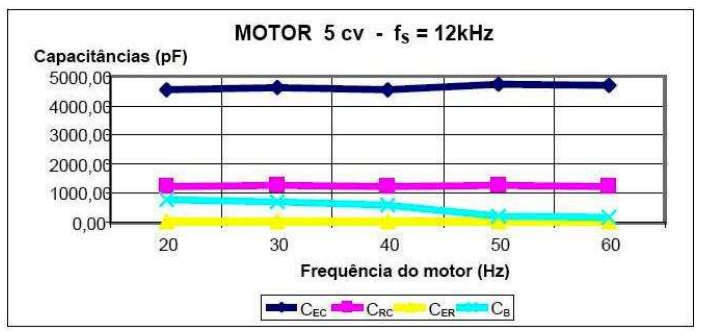

(c)

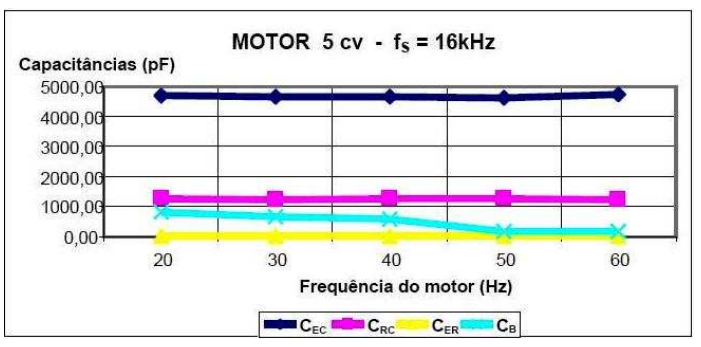

(d)

Figura 14: Capacitâncias parasitas $x$ frequência do motor.

\section{AGRADECIMENTOS}

Os autores agradecem a assistência de Edson Oshiro nas montagens em laboratório e a SEW Eurodrive, na pessoa de Cleber Ruiz, pela doação do Inversor MLP de 5 cv.

\section{REFERÊNCIAS}

Adabi, J., Zare, F., Ledwich, G. and Ghosh, A. (2007). Leakage current and common mode voltage issues in modern ac drive systems, Australasian Universities Power

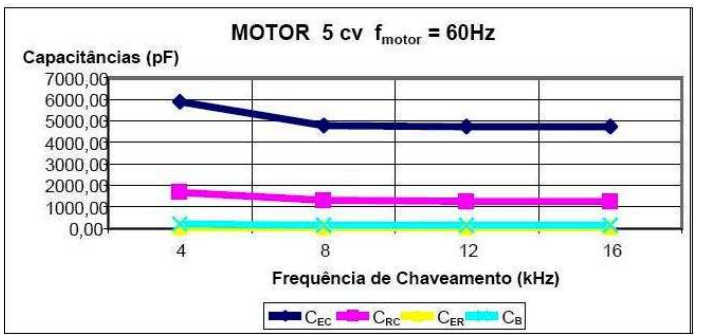

Figura 15: Capacitâncias em função da frequência de chaveamento.

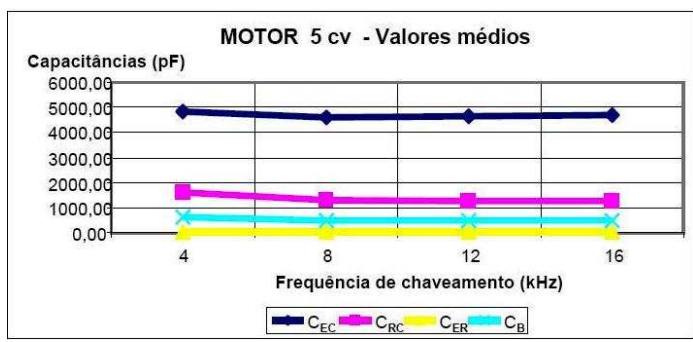

Figura 16: Valores médios das capacitâncias para o motor de $5 \mathrm{cv}$.

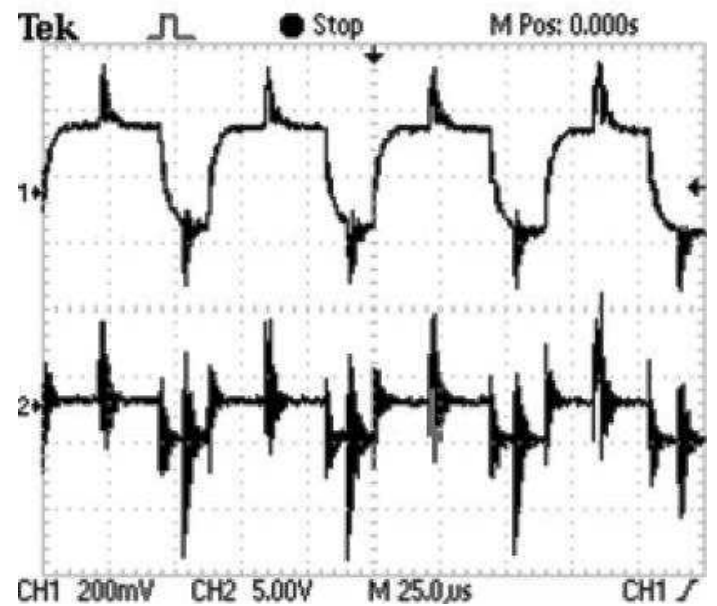

(a)

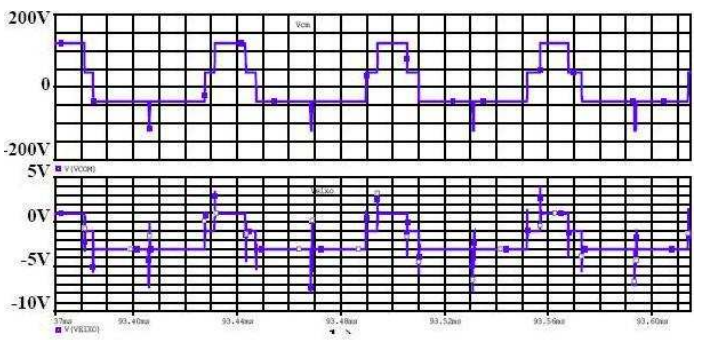

(b)

Figura 17: $\mathrm{V}_{\mathrm{CM}}-\mathrm{Ch}_{1} ; \mathrm{V}_{\text {EIXO }}-\mathrm{Ch}_{2}$. a) medido, b) simulado. 


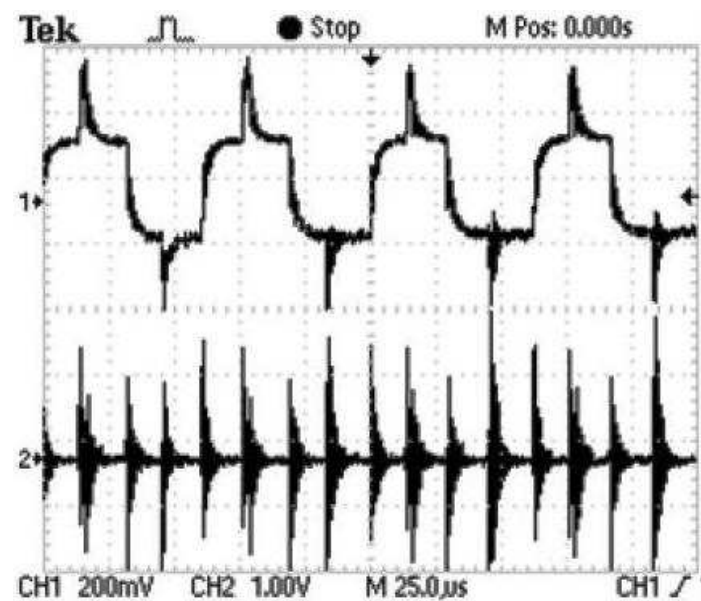

(a)

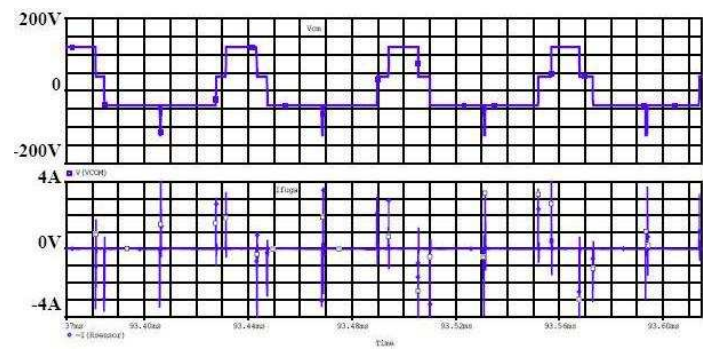

(b)

Figura 18: $\mathrm{V}_{\mathrm{CM}}-\mathrm{Ch}_{1} ; \mathrm{I}_{\mathrm{FUGA}}-\mathrm{Ch}_{2}$. a) medido, b) simulado.

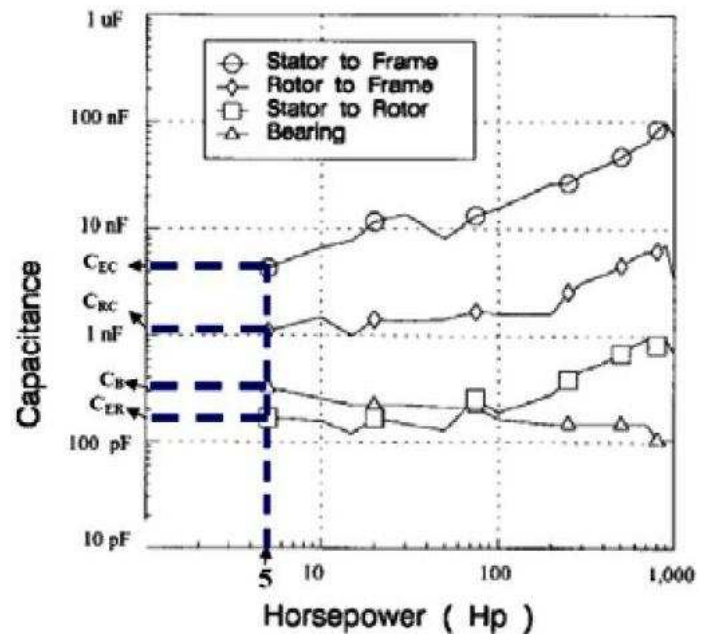

Figura 19: Referência Busse et al. (1996) e Busse et al. (1997).

Engineering Conference, 2007. AUPEC 2007., pp. 1-6.

Akagi, H., Hasegawa, H. and Doumoto, T. (2004). Design and performance of a passive emi filter for use with a voltage-source pwm inverter having sinusoidal output

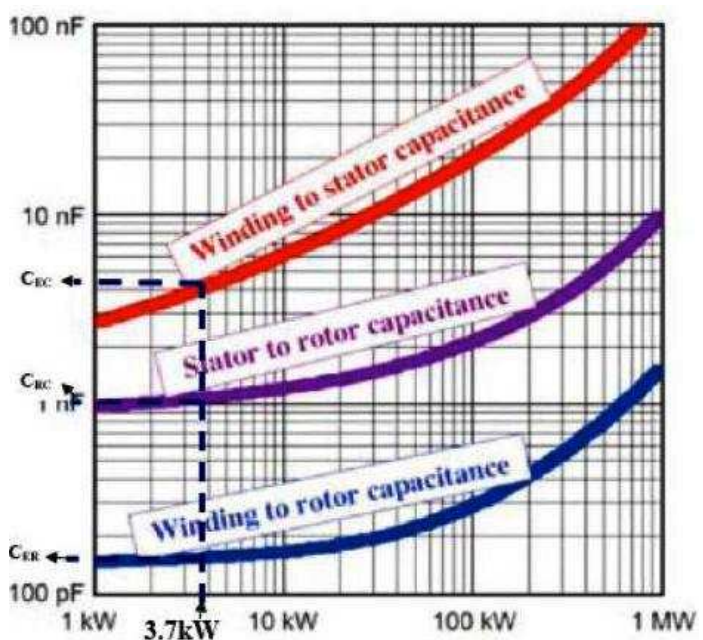

Figura 20: Referência Charoy and Dunand (2007).

voltage and zero common-mode voltage, IEEE Transactions on Power Electronics 19(4): 1069-1076.

Akagi, H. and Tamura, S. (2006). A passive emi filter for eliminating both bearing current and ground leakage current from an inverter-driven motor, IEEE Transactions on Power Electronics 21(5): 1459-1469.

Arnedo, L. and Venkatesan, K. (2002). Pspice simulation for conducted emi and overvoltage investigations in a pwm induction motor drive system, IEEE Workshop on Computers in Power Electronics, 2002. Proceedings., pp. 132-137.

Busse, D., Erdman, J., Kerkman, R., Schlegel, D. and Skibinski, G. (1996). System electrical parameters and their effects on bearing currents, Eleventh Annual Applied Power Electronics Conference and Exposition, 1996. APEC '96. Conference Proceedings 1996., Vol. 2, pp. 570-578.

Busse, D., Erdman, J., Kerkman, R., Schlegel, D. and Skibinski, G. (1997). Bearing currents and their relationship to pwm drives, IEEE Transactions on Power Electronics 12(2): 243-252.

Charoy, A. and Dunand, P. (2007). Bearing current induced by a power drive, Automotive Power Electronics .

Chen, S., Lipo, T. and Fitzgerald, D. (1996). Modeling of motor bearing currents in pwm inverter drives, IEEE Transactions on Industry Applications 32(6): 13651370.

Esmaeli, A., Jiang, B. and Sun, L. (2006). Modeling and suppression of pwm inverter's adverse effects, 1st International Symposium on Systems and Control in Aerospace and Astronautics, 2006. ISSCAA 2006., pp. 1450-1454. 
Fitzgerald, A. E., Kingsley, C. and Umans, S. D. (2003). Electric Machinery, 6 edn, McGraw-Hill Science/Engineering/Math.

Melly, S. (2002). New output filter concept for power drive systems. Product Marketing. SCHAFFNER.

Muetze, A. and Binder, A. (2005). Calculation of motor capacitances for prediction of discharge bearing currents in machines of inverter-based drive systems, IEEE International Conference on Electric Machines and Drives, 2005, pp. 264-270.

Naik, R., Nondahl, T., Melfi, M., Schiferl, R. and Wang, J.S. (2003). Circuit model for shaft voltage prediction in induction motors fed by pwm-based ac drives, IEEE Transactions on Industry Applications 39(5): 12941299.

NBR 05383-1, . (2002). NBR 05383-1 - 2002 - máquinas elétricas girantes - parte 1 - motores de indução trifásicos - ensaios.

Riehl, R. (2010). Uma Metodologia para Determinação das Capacitâncias Parasitas de Motores de Indução Trifásicos de Rotor em Gaiola, PhD thesis, Faculdade de Engenharia Elétrica e de Computação - UNICAMP.

\section{APENDICE A}

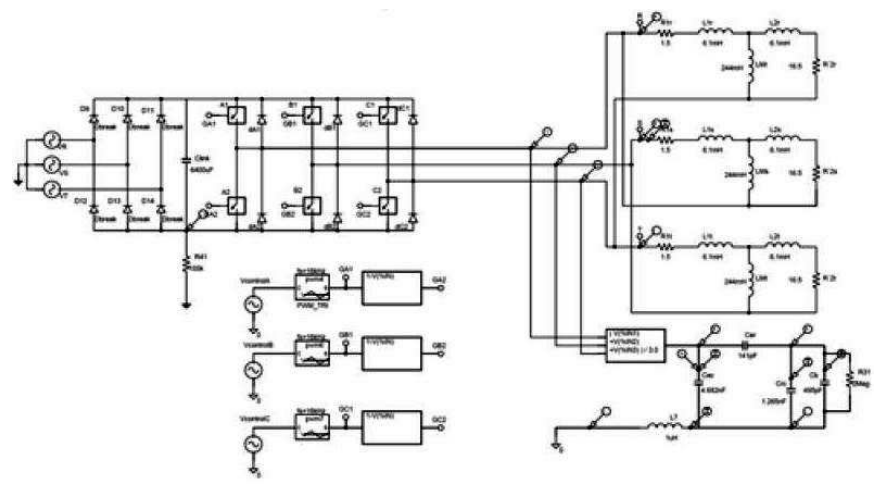

Figura A.1: Circuito esquemático utilizado para simulação no Pspice. 